\title{
Similar effects of different threats on perceptual processes
}

\author{
MARK G. EBERHAGE, DARLENE POLEK, and MICHAEL T. HYNAN \\ University of Wisconsin, Milwaukee, Wisconsin
}

\begin{abstract}
This study was conducted to determine whether repressors and sensitizers would show differential visual recognition in two types of threatening situations. The manipulations of threat were a factorial combination of neutral versus sexual materials and success versus failure feedback. Sixty-four female subjects were classified as either repressors or sensitizers using the Manifest Anxiety Scale. Subjects learned picture-nonsense word paired associates with either neutral (outdoor scenery) or threatening (nude male) pictures as stimuli. Subsequently, subjects were given false feedback (of success or failure) regarding their rate of learning. Subjects were then given a perceptual task which involved recognition of the previous nonsense words. There were no differences in recognition between repressors and sensitizers in the success condition, and when the nonsense words had been paired with neutral pictures. Repressors did take longer than sensitizers to recognize words after the failure experience. Repressors also look longer to recognize the words associated with the threatening pictures. The results support the operation of a generalized perceptual defensiveness in repressors. However, sensitizers showed no indications of perceptual vigilance.
\end{abstract}

Theoretically, defense and vigilance are construed as different perceptual reactions utilized by people responding.to potentially anxiety-producing stimuli. Defense results in an inhibition of perception; vigilance enhances perception (Bruner \& Postman, 1947; Erdelyi, 1974). A particular individual's cognitive style of either vigilance or defense is presumably determined largely by the dominant needs, attitudes, values, and psychodynamic defenses of that individual (Byrne, 1964; Erdelyi, 1974). This cognitive style has been regarded as a stable dimension of personality often referred to as repressionsensitization (Byrne, 1964). Repressors are perceptually defensive; sensitizers are perceptually vigilant.

Proponents of this "new look" have argued that these perceptual processes occur unconsciously (Bruner \& Postman, 1947; Erdelyi, 1974; Lazarus \& McCleary, 1951; McGinnies, 1949). Others have questioned this position. For example, Howes and Solomon (1950) suggested that the higher visual recognition threshold for threatening words reported by McGinnies (1949) was an artifact of the low frequency of use of threatening words. Specifically, Howes and Solomon argued that subjects took longer to recognize threatening words (relative to neutral words) not because the words were threatening, but because the subjects were less familiar with threatening words than with neutral words.

Howes and Solomon (1950) also offered an alternative interpretaion to explain why subjects took longer to recog-

Mark G. Eberhage is now at the University of Missouri, Kansas City, Missouri. Darlene Polek is now at the University of South Carolina, Columbia, South Carolina. Reprint requests should be sent to Michael T. Hynan, Psychology Department, University of Wisconsin-Milwaukee, P.O. Box 413, Milwaukee, WI 53201. nize threatening materials than neutral materials. Howes and Solomon proposed that subjects consciously withheld the verbal report of a threatening word after recognition because of embarrassment. This conscious inhibition against saying socially unacceptable words has also been reported by others (Cowen \& Beier, 1950). In light of these potential artifacts, other researchers have investigated whether perceptual defense or vigilance would occur when word familiarity was controlled and when conscious inhibition was unnecessary.

Hutt and Anderson (1967) paired nonsense syllables with neutral and threatening words. When these nonsense syllables were later presented alone tachistoscopically, repressors took significantly longer to recognize syllables previously paired with threatening words than those paired with neutral words. However, sensitizers showed no difference in recognition between the threatening and neutral conditions.

Similarly, Tempone (1962) gave repressors and sensitizers the task of solving anagrams of neutral words. Then, subjects were told that they either had succeeded or had failed the anagram task. Subsequently, subjects were exposed to the correct anagram solutions and other control words in a tachistoscopic recognition task. Tempone found an interaction of repression-sensitization with the success-failure manipulation. In the success condition repressors and sensitizers performed comparably in recognizing the anagram solutions. In the failure condition sensitizers recognized the anagram solutions at significantly lower thresholds than did repressors. For the control words, there was no relationship between perceptual mode and the success-failure manipulation.

This experiment is a replication of the basic three-factor design in the Tempone (1962) study, using different 
threatening materials and a different recognition task. In the present study repressors and sensitizers first engaged in a paired-associate learning task where the responses were nonsense words and the stimuli were either neutral or threatening pictures. Then subjects were given either success or failure feedback regarding their performance on the paired-associate task. Subsequently, subjects were tested for visual recognition of the nonsense words using a page-turning procedure.

We hypothesized that the data would show a triple interaction. On the basis of Tempone's (1962) data, we expected that sensitizers would recognize the words associated with threatening pictures faster than repressors only in the failure condition. Also, we expected no significant difference between sensitizers and repressors in recognizing the threatening nonsense words in the success condition. We also expected that there would be no difference between repressors and sensitizers in recognition of words associated with neutral pictures.

\section{METHOD}

\section{Subjects}

Sixty-four female students in introductory and intermediate psychology courses at the University of Wisconsin-Milwaukee participated for extra course credit.

\section{Materials}

The original version of the Taylor (1953) Manifest Anxiety Scale (MAS) was used as an index of repression-sensitization. The MAS was used rather than the Byrne Repression-Sensitization Scale for ease of administration. The MAS has been reported to have a correlation of 0.91 with the Byrne Repression-Sensitization Scale (Byrne, 1961, 1964).

Five pictures of outdoor scenery were taken from Omni and Newsweek for the neutral pictures. Five pictures of nude males were taken from Playgirl magazine for the threatening pictures. Each picture was affixed to a standard sheet of typing paper. Each of five structurally similar nonsense words (SEBON, ZAREG, BONAK, FAPUR, and PEFOD) were typed on separate sheets of paper. Each word was then randomly paired with both a neutral and a threatening picture. Picture-word pairs were then stapled in booklet form so that the picture was seen first, then its word associate, then the next picture, and so forth. One learning booklet contained the five neutral pairs, and the other learning booklet contained the five threatening pairs.

The recognition task used materials similar to those described by White (1949) and Cowen and Beier (1950). Each nonsense word was typed onto ten $21.6 \times 14.0-\mathrm{cm}$ sheets of paper with nine pages of carbon paper interleaved. The carbon paper was removed, after which the original and copies were ordered from most blurred to clearest and stapled in a booklet form. A separate test booklet was made for each of the five words.

Sessions took place in a small experimental room with two chairs on opposite sides of a table.

\section{Procedure}

A male experimenter instructed each subject that she had $5 \mathrm{~min}$ to complete the MAS. The MAS was then scored by a female experimenter seated in an adjacent hallway. Subjects were designated as repressors or sensitizers using a median split of raw scores (median =14). Each subject then carried out the paired-associate study task in the presence of the male experimenter. Each subject was given $3 \mathrm{~min}$ to page through either the neutral or the threatening booklet at her own pace. Then the male experimenter displayed each stimulus picture for $5 \mathrm{sec}$, requesting that the subject say aloud the nonsense word that had been paired with it.
The male experimenter then left the room, and the female experimenter entered the room $1 \mathrm{~min}$ later announcing that the subject had done either "better than most people" or "worse than most people" on the learning task. Next, subjects were presented with the booklets of blurred to clear copies of each nonsense word. Subjects were also told that a nonsense word learned in the first task would appear in each booklet. Subjects were instructed to go through each booklet one page at a time, turning a page every $3 \mathrm{sec}$ when the experimenter said "Turn." Subjects were encouraged to say the word aloud or spell the word as soon as they could guess what it was. Subjects were debriefed after the recognition task.

\section{RESULTS}

Data for both the learning and the recognition tasks were analyzed using a 2 (repression-sensitization) $\times 2$ (neutralthreatening) $\times 2$ (success-failure) analysis of variance.

\section{Learning}

There were no significant effects of any of the independent variables upon the number of paired associates learned. Overall, subjects recalled an average of 2.3 of the five nonsense words.

\section{Recognition}

Overall, repressors took more page turns (mean $=4.4)$ than did sensitizers (mean $=3.8$ ) to identify the nonsense words in the recognition task $[F(1,56)=9.6, p<.01]$. There were no significant main effects for the neutralthreat manipulation or the success-failure manipulation. There was a significant interaction of repressionsensitization with the success-failure manipulation $[F(1,56)=4.9, p<.05]$. There was also a significant interaction of repression-sensitization with the neutralthreat manipulation $[F(1,56)=8.5, p<.01]$. The other two-way interaction and the three-way interaction were not significant.

Figure 1 illustrates both significant two-way interactions. The left panel of Figure 1 shows that repressors and sensitizers performed comparably in the success condition. After the failure experience, however, repressors took more page turns than sensitizers to recognize the nonsense words $[F(1,15)=15.6, p<.01]$. The right panel

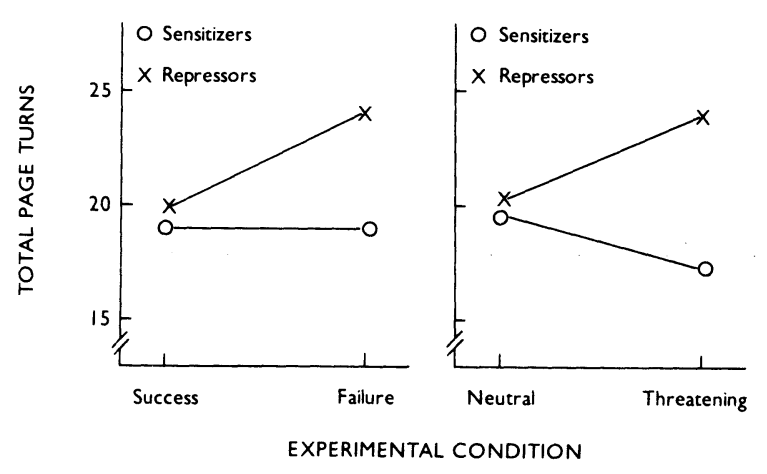

Figure 1. Mean total page turns for the five booklets (maximum $=50)$. 
of Figure 1 shows that repressors and sensitizers were comparable in recognizing words that had been associated with the neutral pictures. Repressors, however, took longer than sensitizers to recognize the words that had been associated with the threatening pictures $[F(1,15)=$ $17.4, p<.01]$. In both interactions it is important to note that there were no significant differences in recognition by sensitizers across all experimental conditions. Repressors, however, did take longer to recognize words associated with failure than those associated with success $[F(1,15)=5.7, p<.05]$. Repressors also took longer to recognize words associated with threatening pictures as opposed to neutral pictures $[\mathrm{F}(1,15)=5.3, \mathrm{p}<.05]$.

\section{DISCUSSION}

The results support the operation of perceptually defensive processes when repressors are in threatening situations. Indeed, the process of perceptual defense in this study was more generalized than initially predicted. We had hypothesized that perceptual effects would occur only when repressors had experienced the combination of threatening situations (threatening pictures plus failure feedback). Instead, either threatening situation was in itself sufficient to produce delayed recognition in repressors.

In contrast to the support for perceptual defense, there was little indication of perceptual vigilance by sensitizers. Sensitizers recognized the nonsense words after comparable page turns in all conditions, threatening and nonthreatening. This outcome can be interpreted in a number of ways. One possibility is that personality determinants of perception may be limited only to delays in recognition, not to vigilance. Such a pattern is consistent with other studies (Hutt \& Anderson, 1967; Tempone, 1962). However, the number of studies which do demonstrate vigilance makes this conclusion problematic (Erdelyi, 1974).

A second interpretation of the pattern of results would be that the amount of threat in the experiment was insufficient for vigilance to occur. This interpretation has the implication that perceptual defense and vigilance have different thresholds of occurrence. It is possible that viewing pictures of nude males and learning that one has failed are sufficiently threatening to produce defense in repressors, but not threatening enough to produce vigilance in sensitizers. This notion is consistent with work reported by Bruner and Postman (1974), who found that a critical degree of emotionality was necessary for the occurrence of vigilance.

A third interpretation (one which we favor) is that there was insufficient power in the study to demonstrate vigilance. The two key conditions in this experiment were the ones in which the two personality types (repressors and sensitizers) had experienced failure after viewing nude pictures. These two conditions did produce the extremes of recognition found in the study (mean=5.3 page turns/word for repressors vs. mean $=3.5$ pages turns/word for sensitizers). With additional subjects the data analyses may well have shown a process of sensitization within the context of the predicted three-way interaction.

The results do clearly support one aspect of repression-sensitization theory (Byrne, 1964). Repressors had delayed conscious recognition in the threatening situations. This outcome occurred in the context of a study designed to control for possible confounding variables. Specifically, subjects learned the picture-word associates at an equivalent rate across conditions; subjects had equivalent familiarity with the nonsense words used in the recognition task; and subjects had no apparent need to consciously suppress saying the nonsense words. Future research should use male subjects with similar procedures in order to determine whether there are sex differences in perceptual defense. The use of other threatening conditions would also help to establish the generality of perceptual determinants of perception.

\section{REFERENCES}

Bruner, J. S., \& Postman, L. (1947). Emotional selectivity in perception and reaction. Journal of Personality, 16, 69-77.

BYRNE, D. (1961). The repression-sensitization scale: Rationale, reliability, and validity. Journal of Personality, 29, 334-349.

BYRNE, D. (1964). Repression-sensitization as a dimension of personality. In B. A. Maher (Ed.), Progress in experimental personality (pp. 169-191). New York: Academic Press.

COWEN, E., \& BEIER, E. (1950). The influence of "threat-expectancy" on perception. Journal of Personality, 19, 85-94.

ERDELYi, M. H. (1974). A new look at the new look: Percpetual defense and vigilance. Psychological Review, 81, 1-25.

Howes, D. H., \& Solomon, R. L. (1950). A note on McGinnies' "Emotionality and perceptual defense." Psychological Review, 57, 229-234.

Hutt, L. D., \& Anderson, J. P. (1967). Perceptual defense and vigilance: Prediction from the Byrne scale of repression-sensitization. Psychonomic Science, 9, 473-474.

Lazarus, R. S., \& McCleary, R. A. (1951). Autonomic discrimination without awareness: A study of subception. Psychological Review, 58, 113-122.

McGinnies, E. (1949). Emotionality and perceptual defense. Psychological Review, 56, 244-251.

TAYLOR, J. A. (1953). A personality scale of manifest anxiety. Journal of Abnormal \& Social Psychology, 48, 285-290.

TEMPONE, V. J. (1962). Differential thresholds of repressors and sensitizers as a function of a success or failure experience. Unpublished doctoral dissertation, University of Texas, Austin.

White, M. A. (1949). A study of schizophrenic language. Journal of Abnormal Social Psychology, 44, 61-74.

(Manuscript received for publication July 9, 1985.) 